Chapter 13

\title{
Carbon Nanotubes for Use in Medicine: Potentials and Limitations
}

\author{
Wei Shao, Paul Arghya, Mai Yiyong, \\ Laetitia Rodes and Satya Prakash \\ Additional information is available at the end of the chapter \\ http://dx.doi.org/10.5772/51785
}

\section{Introduction}

Structurally, Carbon nanotubes (CNTs) can be viewed as wrapped from graphene sheets. Single-walled carbon nanotubes (SWNTs) have one layer of graphene sheet, whereas, the multiwalled carbon nanotubes (MWNTs) contain multi layers of graphene sheets. The wellordered molecular structure brings CNTs many remarkable physical properties, such as, excellent mechanic strength, ultrahigh surface area, high aspect ratio, distinct optical properties [1], and excellent electrical conductivity [2]. In last decade, CNTs are intensively explored for in-vitro and in-vivo delivery of therapeutics, which was inspired by an important finding that CNTs can penetrate cells by themselves without apparent cytotoxic effect to the cells [3]. The high aspect ratio makes CNTs outstanding from other types of round nanoparticles in that the needle-like CNTs allow loading large quantities of payloads along the longitude of tubes without affecting their cell penetration capability. With the adequate loading capacity, the CNTs can carry multifunctional therapeutics, including drugs, genes and targeting molecules, into one cell to exert multi-valence effects. In the other side, owing to the ultrahigh surface area along with the strong mechanical properties and electrically conductive nature, CNTs are excellent material for nanoscaffolds and three dimensional nanocomposites. In recent year, CNT-based devices have been successfully utilized in tissue engineering and stem cell based therapeutic applications, including myocardial therapy, bone formation, muscle and neuronal regeneration. Furthermore, owing to the distinct optical properties of CNTs, such as, high absorption in the near-infrared (NIR) range, photoluminescence, and strong Raman shift [4], CNTs are excellent agents for biology detection and imaging. Combined with high surface area of CNTs for attaching molecular recognition molecules, CNT-based, targeted nanodevices have been developed for selective imaging 
and sensing. There are many areas where CNTs are extremely useful. Given the scope in this chapter, We describe strategies for preparation of CNTs for their use in medicine. Specifically, we focus and highlight the important biomedical applications of CNTs in the field of drug delivery, gene delivery, stem cell therapy, thermal therapy, biological detection and imaging (figure 1). The methods for formulating CNT-based therapeutics to suit different routes of drug administration are also described. The limitations with emphasis on toxicity and over all future directions are discussed.

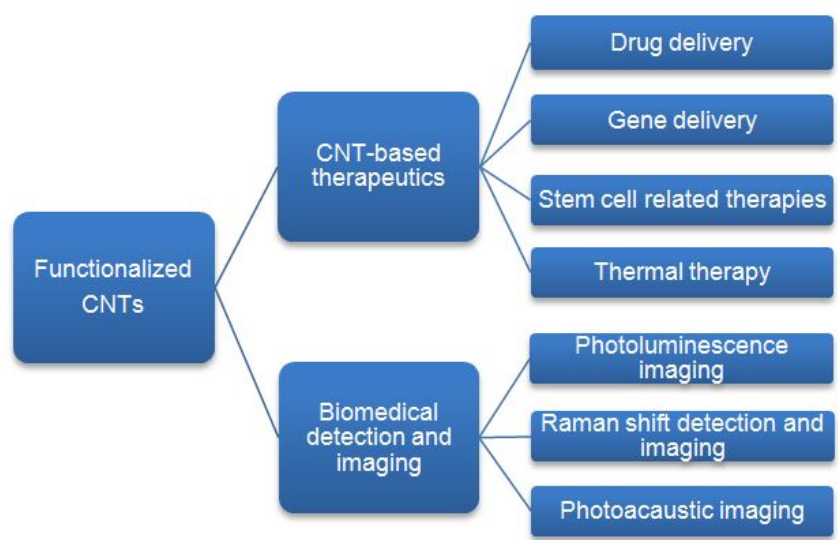

Figure 1. Functionalized CNTs in major biomedical applications.

\section{Preparation of CNTs for use in medicine}

Raw CNTs, persisting metallic nature, are highly hydrophobic. Therefore, surface modification of CNTs, or CNT functionalization, so as to disperse them into aqueous solutions becomes a key step for their biomedical applications. The CNT modification methods are involved in non-covalent and covalent strategies. The non-covalent modification utilizes the hydrophobic nature of CNTs, especially, $\pi-\pi$ interactions for coating of amphiphilic molecules. The covalent modification generates chemical bonds on carbon atoms on CNT surface via chemical reactions followed by further conjugation of hydrophilic organic molecules or polymers rendering CNTs better solubility. These modifications not only offer CNTs water solubility, but also produce functional moieties that enable linking of therapeutic agents, such as genes, drugs, and recognition molecules for biomedical applications. 


\subsection{Non-covalent modification of carbon nanotube surface}

The non-covalent modification approaches typically use amphiphilic molecules ranged from small molecules to polymers. The amphiphilic molecules associate with CNTs by either adsorbing onto or wrapping the CNTs [5]. The non-covalent modifications of CNTs are easy to perform. The process is only involved in sonication of CNTs with amphiphilic molecules in solvent at room temperature. Since it is a mild condition, CNTs molecular structure is not affected, and therefore their optical and electrical conductive properties are conserved.

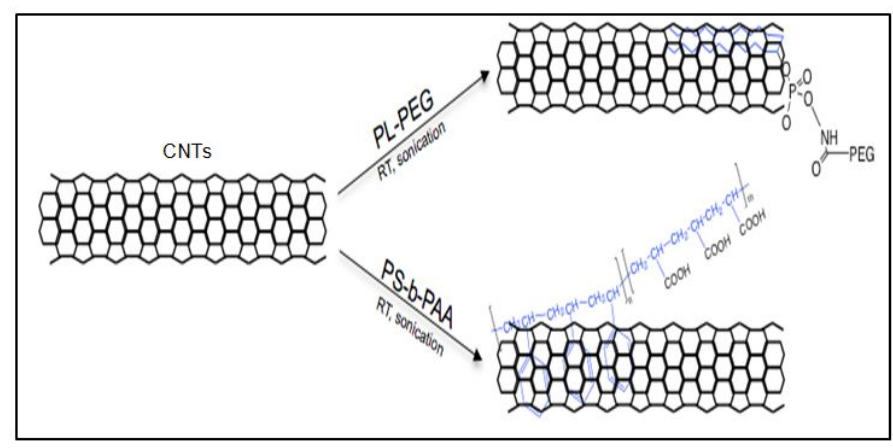

Figure 2. Schematic representation of adsorption of amphiphilic molecules onto carbon nanotube surface by $\pi-\pi$ stacking and other hydrophobic interactions. Abbreviations: PL-PEG, phospholipid-polyethylene glycol; PS-b-PAA, polystyrene-block-polyacrylic acid.

Adsorption of amphiphilic molecules, such as surfactants, amphiphilic copolymers or others, onto CNT surfaces is one of the simplest and most effective way to disperse CNTs without destruction of their $s p^{2}$ hybridization [5]. The hydrophilic portions of surfactants interact with the polar solvent molecules, whereas, the hydrophobic portions adsorb onto the nanotube surface $[5,6]$. The dispersity depends strongly on the length of the hydrophobic regions and the types of hydrophilic groups in the amphiphilic molecule. For example, surfactants with ionic hydrophilic head groups, such as sodiumdodecylsulfate (SDS) [7] or cetyltrimethyl ammonium bromide (CTAB) [8,9], can stabilize a nanotube by electrostatic repulsion between micellar domains [7]. Nonionic surfactants, such as Triton X-100 [8], disperse CNTs mainly by forming a large solvation shell around a nanotube [8]. Figure 2 illustrates the manor of adsorbing amphiphilic molecules onto CNT surfaces, in which, hydrophobic alkyl chains or aromatic rings lay flat on graphitic tube surfaces. For example, an synthetic biocompatible lipid-polymer conjugate, phospholipid-polyethylene glycol (PLPEG) has been applied for surface modification of CNTs, which gives rise to a variety of biomedical applications ranged from drug delivery, biomedical imaging, detection and biosensors [10]. The ionic surfactants, particularly those based on alkyl-substituted imidazolium cationic surfactants [11], can effectively disperse CNTs in organic or aqueous media by the counter anion $[12,13]$. Polyaromatic derivatives carrying hydrophilic moieties can also effectively disperse CNTs in aqueous media by forming specific directional $\pi-\pi$ stacking 
with the graphitic surfaces of nanotubes $[6,14]$. In this context, pyrene, a polyaromatic molecule, demonstrated a high affinity toward CNT surfaces [6]. Interactions of the polyaromaticmoitie of pyrene with CNTs are strong enough to be irreversible, and therefore, the pyrene derivatives are used to anchor proteins or biomolecules on nanotube surface [6, 14, 15]. Other classes of polyaromatic molecules, such as substituted anthracenes, heterocyclic polyaromaticporphyrins [16] and phthalocyanines [17], disperse CNTs via the same mechanism.

The polymers containing hydrophobic backbone and hydrophilic side groups, eg. poly[p$\{2,5$-bis(3-propoxysulfonic acid sodium salt)\}phenylene] ethynylene (PPES), can effectively disperse CNTs in water, in which, the strong $\pi-\pi$ interactions between CNTs and aromatic backbone of the polymers drive the wrapping of CNTs, and the water-soluble side groups impart solubility of CNTs in water [18]. DNA and siRNA can disperse CNTs by wrapping. DNA or siRNA are made of hydrophobic bases and alternative hydrophilic phosphates and riboses. Such structure facilitates CNT dispersion by the bases wrapping to CNTs and the hydrophilic sugar-phosphate groups extending to water phase [19].

\subsection{Covalent modification of CNT surface}

The covalent modification, namely the chemical modification of CNTs is an emerging area in materials science. Among the various strategies, the most common ones are:

i. esterification and amidation of oxidized CNTs,

ii. generation of functional groups on CNT sidewalls by cycloaddition reactions.

Oxidation of CNTs is a purification method for raw CNTs. Oxidation of CNTs is carried out by reflexing raw CNTs in strong acidic media, e.g. $\mathrm{HNO}_{3} / \mathrm{H}_{2} \mathrm{SO}_{4}$. Under this condition, the end caps of the CNTs are opened, and carboxylic groups are formed at these ends caps and at some defect sites on nanotube sidewalls (Figure 3a) [20]. The carboxylic groups provide opportunities for further derivatization of the CNTs through esterification or amidation reactions. For example, some organic molecules with amine groups can be directly condensed with the carboxylic groups present on the surface of the CNTs [6, 14]. Alternatively, the carboxyl moieties can be activated with thionyl chloride and subsequent react with amine groups (Figure 3b) $[6,14]$. These reactions are widely applied for conjugation of water-soluble organic molecules, hydrophilic polymers, nucleic acid (DNA or RNA), or peptides to the oxidized CNTs, which result in multifunctional CNTs $[6,14]$. In most cases, the length of nanotubes is often shortened [20], but the electronic properties of such functionalized CNTs remain intact. Oxidation reaction only generates carboxyl groups on cap ends and defect sites on CNTs. To generate chemical bonds on sidewall and cap ends of CNTs, cycloaddition reactions are used [21](Figure 4). Cycloaddition reaction is a very powerful methodology, in which the 1,3-dipolar cycloaddition of azomethineylides can easily attach a large amount of pyrrolidine rings on sidewalls of nanotubes. Thus, the resulting functionalized CNTs are highly soluble in water [22]. In addition, pyrrolidine ring can be substituted with many functional groups for different applications. 


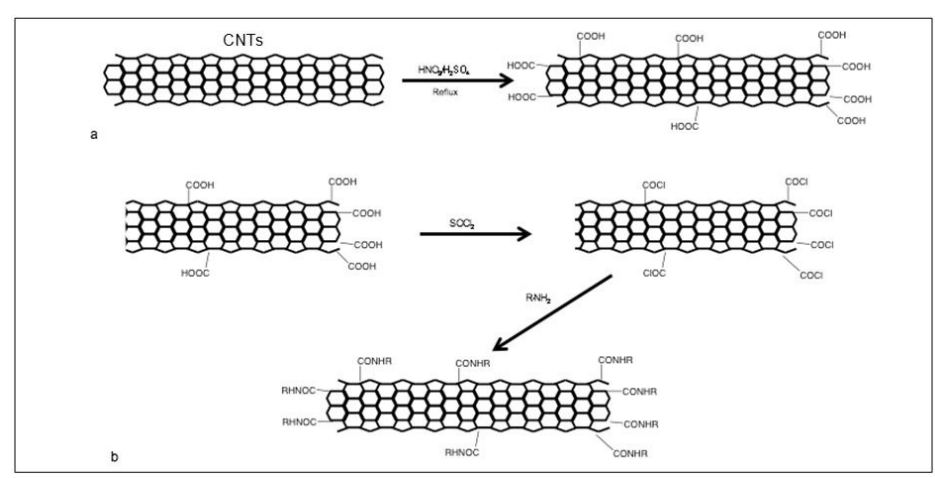

Figure 3. Covalent modification of carbon nanotubesby a) Oxidation reaction of cap end of CNTs and b) further attaching hydrophilic molecules by amidation reactions.

In contrast to non-covalent surface modifications, which do not locally disrupt $s p^{2}$ hybridization, or create defects, the covalent surface modifications disrupt CNT $s p^{2}$-conjugated structures and therefore, could affect the electronic and optical performances [5].

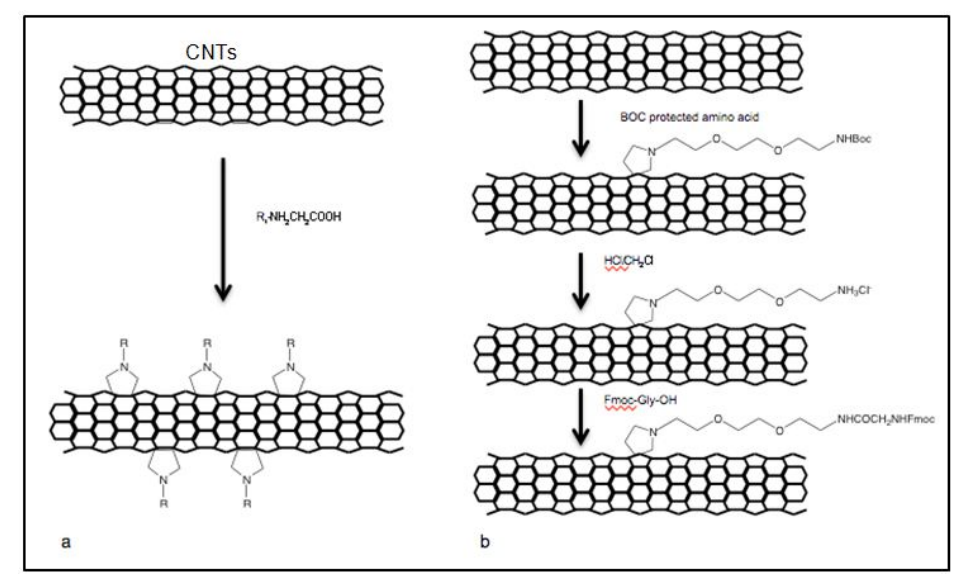

Figure 4. Sidewall covalent modification of carbon nanotubes a) a general scheme of 1,3-dipolar cycloaddition reaction b) preparation of amino-functionalized CNTs by 1,3-dipolar cycloaddition.

\section{Carbon nanotube based therapeutics}

\subsection{Carbon nanotubes for chemotherapy drug delivery}

Cancer is one of the most common causes of death worldwide. Chemotherapy in addition to the surgical removal of tumors is a conventional treatment for cancers. However, the effec- 
tiveness of chemotherapy drugs is often limited by the toxicity to other tissues in the body. This is because most chemotherapy drugs do not specifically kill cancer cells, they act to kill all cells undergoing fast division. Nanoparticles have been applied to drug delivery and showed improved drug efficiency and reduced off-target tissue toxicity due to accumulation in tumor tissues. Nanoparticles target tumor tissues by two mechanisms: passive targeting and active targeting. As fast growing tissues, tumors display enhanced vascular permeability due to high demand for nutrients and possible oxygen. The features of the leaky vasculature are employed for delivery of nanoparticle drugs since the size of nanoparticle allows them to accumulate in tumor tissues [23]. The phenomenon is termed as tumor-selective enhanced permeability and retention (EPR) effect. More efficient tumor targeting can be achieved through active targeting approaches, in which, targeting molecules can recognize tumor biomarkers on cancer cell surface. The properties of CNTs are beneficial for cancer drug delivery, firstly, like other nanoparticles, the size of functionalized CNTs is preferable for accumulation in tumor tissues; secondly, CNTs contain ultrahigh surface area of CNTs facilitate loading of drugs and targeting molecules; thirdly, the hydrophobic benzene ring structure of CNTs can be used for loading drugs that contain benzene ring structure, eg. doxorubicin (DOX), epirubicin (EPI), and daunorubicin (DAU).

\section{Preparation of tumor-targeted devices using carbon nanotubes}

A range of tumor targeting molecules has been discovered, including tumor specific antibodies, peptides, and others. Antibodies have been developed to specifically binding to biomarkers on cancer cell surface, eg, Trastuzumab recognizes Human Epidermal Growth Factor Receptor 2 (HER-2) positive cancer cells[24] and anti-CD20 for CD20 biomarker on B cell lymphoma [25]. These antibodies have therapeutic effects on their own, and can also serve as tumor targeting probes. Alpha V beta $3(\alpha \mathrm{v} \beta 3)$ integrin is a heterodimerictransmembrane glycoprotein found on a variety of tumor cells, including osteosarcomas, neuroblastomas, glioblastomas, melanomas, lung, breast, prostate cancers. $\alpha v \beta 3$ ) integrin is a wellrecognized target for cancers. The amino acid sequence of Arg-Gly-Asp (RGD) is identified to be responsible for tight binding to $\alpha \mathrm{v} \beta 3$ integrin, which leads to the development of short tumor targeting peptide RGD [26]. Similar to RGD, another type of peptide contains Asn-Gly-Asp (NGR) triad that binds to the endothelium cells on neoangiogenic vessels. NGR-tagged delivery systems have been developed to deliver cytokines, nanoparticles, and imaging agents to tumor blood vessels [27]. Folic acid, a small molecule vitamin, binds to folate receptor overexpressed in a variety of cancer cells, including breast, colon, renal and lung tumors[28]. As described in section 2 of this chapter, a variety of chemical and physical methods have been developed for functionalization of CNTs. The above listed tumor targeting molecules are mostly proteins or peptides, which contains sulfhydryl groups that can be easily conjugated to amino-functionalized CNTs [14] using heterbifunctional linker molecules that contain NHS ester on one end and Maleimide on the other end [29]. These conjugation reactions are usually carried out under mild conditions [10]. Thus, the molecular structure of CNTs is not disturbed, and therefore, the optical properties are preserved. The CNT-based targeted devices developed by this methods are good for potential tumor detection and imaging applications. To date, all above-mentioned tumor-targeting strategies have 
been applied for construction of CNT-based, which will be discussed separate in the following sub-sections.

\section{Targeted delivery of chemotherapy drugs by physically absorbed on carbon nanotubes}

Supermolecular benzene ring structure of CNTs affords surprisingly high degree of aromatic molecules by $\pi-\pi$ stacking. DOX, an important chemotherapeutic agent, has been efficiently loaded onto SWNTs-PL-PEG for tumor-targeted delivery [30, 31]. Binding to and release of drug molecules from nanotubes could be controlled by adjusting $\mathrm{pH}$.The appropriate diameter of nanotube for drug loading was used because the strength of $\pi-\pi$ stacking of aromatic molecules was dependent on nanotube diameter. In-vivo study with SWNTsPL-PEG/DOX showed significantly enhanced therapeutic efficacy in a murine breast cancer model [30]. With further attaching tumor targeting molecules, eg. folic acid (FA) or RGD, the targeted SWNTs-DOX could more effectively inhibit the growth of cancer cells in-vitro and in-vivo [31-34]. Similar physical absorption method was applied for drug DAU using SWNTs, in which, sgc8c Aptamer was used to target leukemia biomarker protein tyrosine kinase-7 [33]. It has been shown that Aptamer-SWNTs-DAU was able to selectively target leukemia cells. The release of DAU was $\mathrm{pH}$-dependent.Other hydrophobic drug molecules, such as paclitaxel (PTX), docetaxel (DTX), can also be absorbed on CNTs surface for delivery $[32,35]$, however, their loading efficiency and stability were much lower due to their comparatively bulky structure.

\section{Targeted delivery of chemotherapy drugs by covalently linked to functionalized carbon nanotubes}

Non-aromatic small molecule drugs can be chemically conjugated to CNTs for delivery. However, the drugmolecules have to be released from the CNTs to take effect, so the linkages between the drugs and CNTs have to be cleavable. Preferably, the active drugs are released inside of the target cells to reduce toxic effect to the neighbouring healthy cells. The common linkers that are used for drug delivery include ester, peptide, and disulfide bonds. These linkers can be cleaved by the 7enzymes present in the routes of delivery. Specifically designed linkers allow controlled release of drug into desired sites. For example, drug cisplatin has been conjugated directly to oxidized SWNTs via a peptide linker [36]. This specific peptide linkage has been shown tobe selectively cleaved by proteases overexpressed in tumor cells. Further conjugation of epidermal growth factor (EGF), a growth factor that selective binding to EGF receptor overexpressed on cancer cells, to SWNTs-cisplatin led to more efficient tumor inhibition compared to both free cisplatin and non-targeted SWNTscisplatin [36]. Alternative to conjugation of drugs to CNTs directly, drugs can also be conjugated to the molecules, eg. polymers, that are used to disperse CNTs. The end functional groups in the polymers are used for drug linkage. This method is very useful for delivery of bulky, hydrophobic drug molecules. In one example, SWNTs was dispersed using a biocompatible polymer PL-PEG-NH ${ }_{2}[10]$ and drug PTX was conjugated to SWNT-PL-PEG-NH $\mathrm{N}_{2}$ via via ester bonding for delivery [37]. PTX is one of the most important drugs for metastatic breast cancer. However, currently available formulations for PTX have to be infused intravenously over long periods of time due to the side effects. In addition, due to poor water solubility of the drug, necessity of use organic solvent, such as Cremophor in clinical formulation Taxol ${ }^{\circledR}$ causes sever side effects and hypersensitivity reactions $[38,39]$. Conju- 
gation of PTX to SWNTs-PL-PEG-NH $\mathrm{NH}_{2}$ enable removing of solvent in delivery. Indeed, the SWNTs-PL-PEG-PTX displayed increased tumor inhibition effect and reduced side effects in a murine breast cancer model compared with Taxol® formulation [37].

\begin{tabular}{lllll}
\hline Drugs & Targeting Moieties & Cancer Biomarkers & Type of CNTs & References \\
\hline Cisplatin & EGF & EGF Receptor & SWNTs & $[36]]$ \\
\hline Daunorubicin & Sgc8c Aptamer & Tyrosine Kinase-7 & SWNTs & {$[33]$} \\
\hline Docetaxel & NGR & Endothelial Cells & SWNTs & {$[32]$} \\
\hline Doxorubicin & Folate /Magnetic & Folate Receptor & MWNTs & {$[34]$} \\
\hline Doxorubicin & RGD & Integrin avß3 & SWNTs & {$[30]$} \\
\hline Doxorubicin & Folate & Folate Receptor & SWNTs & {$[31,40]$} \\
\hline Gemcitabine & Magnetic & $/$ & MWNTs & {$[41]$} \\
\hline Platinum (IV) & Folate & Folate Receptor & SWNTs & {$[42]$}
\end{tabular}

Table 1. . Abbreviations: SWNTs, single walled carbon nanotubes; MWNTs, multiwalled carbon nanotubes, EGF, epidermal growth factor; RGD, peptide with arginine-glycine-aspartate sequence; NGR, peptide with asparagineglycine - arginine sequence; Sgc8c, oligonucleotide sequence.

\subsection{Carbon nanotubes for gene delivery}

Gene therapy is an important treatment for cancer and other genetic diseases. However, the effects of gene therapy are limited by the efficiencies of transfection and system delivery. Since DNA and siRNA are macromolecules, they cannot pass through cell membrane by themselves, carriers are needed to take them inside of cells to take effects. Structurally, both DNA and siRNA contain anionic phosphodiester backbone that and be complexed with cationic reagents, such as cationic lipids and polymers, etc. For system delivery, the DNA or siRNA can be loaded into cationic nanoparticles made from cationic lipids or polymers [43, 44]. The nanoparticles could protect them from nucleases degradation. Since CNTs are able to penetrate cells [3], they are investigated for gene delivery. Typically, two methods are used for loading nucleic acids to CNTs:

i. electrostatic association with cationic molecule functionalized CNTs $[45,46]$;

ii. chemical conjugation of nucleic acids to functionalized CNTs via cleavable chemical bonds [47];

iii. DNA or siRNA are directly wrap to raw or oxidized CNTs.

Gene delivery using cationic molecule functionalized carbon nanotubes via electrostatic interactions

As discussed early, cationic molecules, such as, ammonium-containing molecules and polyethylene imine (PEI), can be covalently linked to chemically modified CNTs by oxidation or 1,3-cycloadditions reactions[47-50]. In one application, DNA was loaded into CNTs conjugated with ammonium-terminated oligoethylene glycol(CNTs-OEG- $\mathrm{NH}_{3}{ }^{+}$) for delivery [47, 
48]. Using this deliver vehicle, expression of test plasmid pCMV- $\beta$ gal was examined in-vitro.Result showed that the transfection efficiency of CNTs carrier was 5-10 times higher than naked DNA; but, much lower than that of liposome [47]. It has been shown that charge ratio (ammonium groups on CNTs vs phosphate groups of the DNA backbone) is a determination factor for gene expression [48]. In contrast to DNA delivery, the same CNTs carrier for delivery of cyclin A2 siRNA demonstrated pronounced silencing effect in-vitro[51]. Surprisingly, In-vivo delivery of SOCS1 significantly inhibited SOCS1 expression and retarded the tumor growth in murine B16 tumor model [52]. The studies with PEI functionalized CNTs also showed very positive results. PEI is an efficient gene delivery reagent by its own, however, high amount of PEI is toxic to cells. The siRNA delivery by PEI-grafted MWNTs showed improved gene expression to the equivalent amounts of PEI polymer alone but with reduced cytotoxicity $[46,53]$.

Gene delivery by covalently conjugation to carbon nanotubes via cleavable chemical bonds

Alternatively, genes can be conjugated to amphiphilic polymers that are used for non-covalent CNT functionalization [10,54,55]. Incorporation of cleavable chemical bonds facilitates releasing of DNA or siRNA cargos from CNTs in a controlled manner [54]. Thiol-modified DNA or siRNA were covalently conjugated to amino group of SWNT-PL-PEG- $\mathrm{NH}_{2}$ via cleavable disulfide bond [55]. The genes were released by the cleavage of disulfide bonds by thiol digesting enzymes upon cellular internalization of CNT-PL-PEG-siRNA. The CNT-mediated siRNA delivery showed better gene transfection efficiency than liposome-based delivery system in hard-to-transfect human T cells and primary cells lines [54].

Gene delivery by wrapping directly on carbon nanotubes

Nucleic acids, DNA or siRNA, contains alternative amphiphilic motifs, which can be used to disperse CNTs in water. The nucleic acids form helical wrapping around the CNTs with the bases binding to the hydrophobic CNTs and the hydrophilic sugar-phosphate groups extending to the water phase [19]. In this way, DNA or siRNA serves both CNT dispersing agent and the cargo. It has been shown that the siRNA functionalized SWNTs readily enter cells and exerts its biological activity in cell culture [19]. Studies with intratumoralinjection of siRNA functionalized SWNTs showed significantly inhibition effect in-vivo [56].

\subsection{Carbon nanotubes for stem cell related therapies}

There has been an increasing trend in attempts to design and develop different CNT based tools and devices for tissue engineering and stem cell therapy applications. In particular, CNT impregnated nanoscaffolds have shown multiple advantages over currently available scaffolds. This includes its strong mechanical properties, resemblance of structure with collagen fibrils and extracellular matrix and electrically conductive nature. These attributes of the CNT based scaffolds and three dimensional nanocomposites have led to their diverse therapeutic applications in the field of myocardial therapy, bone formation, muscle and neuronal regeneration. These applications are mainly based on one principle and that is to modulate the stem cell growth and differentiation in a more controlled and desirable manner.

Carbon nanotubes for stem cell based heart therapy 
Over the past two decades there has been significant advancement in stem cell therapy to repair and replace damaged tissues, such as heart muscle[57]. This is because of their ability to divide and differentiate into diverse specialized cell types. Recently, there has been increasing body of evidence indicating that the extracellular matrix plays a critical role in stem cell viability, proliferation and differentiation $[58,59]$. Hence, designing a microenvironment prepared from polymeric scaffolds which imitate the physical characteristics of natural biomatrix has been the central strategy in tissue engineering. The emergence of nanomaterials such as nanotubes provide opportunities to design such biocompatible scaffolds for hosting and directing stem cell differentiation [60].

Preliminary studies demonstrate that neonatal rat ventricular myocytes cultured on substrates of multiwall carbon nanotubes can interact with the nanofibres by forming tight contacts and show significantly improved mitotic and chemotactic effects [61]. Moreover, such mode of culture also altered the electrophysiological properties of cardiomyocytes, indicating that $\mathrm{CNts}$ are able to promote cardiomyocyte maturation. Further investigations with a nanocomposite of PLGA:CNF show that cardiomyocyte density increases with greater amounts of CNF in PLGA [62]. The study also showed similar trends with neurons. The immense potential of this technology for myocardial therapy roots from the fact that this cardiac patch can not only promote myocardial cells, but also induce the nerve cell growth that help the cardiac cells to contract. In addition, it also supports endothelial cells that make the inner lining of the blood vessels supplying oxygen to the heart.

\section{Carbon nanotubes for stem cell based bone regeneration}

In order to direct stem cell differentiation towards bone regeneration, there has been increasing interest by the researchers to explore topographical features of the cell culture substrate. Physical factors, such as rigidity of the extracellular environment, can influence stem cell growth and differentiation. Such differentiation of human stem cells can be detected by altering the size of the nanotubes on which the cells are grown [63]. It has been reported that 70- to 100-nm diameter nanotubes can initiate rapid stem cell elongations, which induce cytoskeletal stress and selective differentiation into osteoblast-like cells, offering a promising route for quicker and better recovery, for example, for patients who undergo orthopedic surgery. The group also showed that the differentiated stem cells express osteopontin and osteocalcin, the two important osteogenetic protein markers.

Moreover CNTs are promising materials for nanaoscaffold and implantation purposes due to the fact that CNTs are conductive, have excellent mechanical properties and their nanostructured dimensions mimic the 3D structure of proteins found in extracellular matrices. Their dimensions resembles closely with that of the triple helix of collagen fibrils which can promote for nucleation and growth of hydroxyapatite, the major inorganic component of bone. A newly developed nanocomposite scaffold of CNFs/CNTs has been shown to influence the cell behaviour [64]. In-vitro study demonstrated that, smaller dimension CNFs dispersed in polycarbonate urethane promoted osteoblast adhesion but did not promote the adhesion of fibroblasts, chondrocytes, and smooth muscle cells. But the mechanisms that guide such cell functions are yet to be understood. 
Surface functionalizing the nanotube surface with bone morphogenetic protein-2 (BMP-2) further accelerates chondrogenic and osteogenic differentiation of MSCs [65, 66]. This stimulation is a combined effect of the surface nanoscale geometry of the substrate nanostructures and their BMP-2 coating efficiency. In a similar kind of study, the system also exhibited higher cell proliferation rate, apart from enhanced differentiation [66]. Nanotubes can also be used for extended drug release as has been demonstrated by Hu et al, where drug loaded nanotubes, in combination with multilayers of gelatin and chitosan, have been shown as a new way to use nanotubes as reservoir for storing drugs [67]. The system effectively promoted osteoblastic differentiation of MSCs. Further studies in this direction can be beneficial in order to develop potential bone implants for improved bone osteointegration.

Carbon nanotubes for stem cell based neuronal regeneration

The unique abilities of human embryonic stem cells (hESCs), such as their self-renewal and potency, hold great promise in the field of regenerative medicine and stem cell based therapy. The derivation of neuronal lineages from hESCs holds promise to treat neurological pathologies of the central and peripheral nervous system such as Parkinson's disease, spinal cord injury, multiple sclerosis and glaucoma [68,69]. CNT based substrates have been shown to promote neuronal differentiation [70]. It has also been proposed that neurons grown on a CNT meshwork displayed better signal transmission, due to tight contacts between the CNTs and neural membranes conducible to electrical shortcuts [71]. It was demonstrated that the MSCs and the neurosphere of cortex-derived neural stem cells (NSCs) can grow on the CNT array and both MSCs and NSCs interacted with the aligned CNTs. The results suggest that CNTs assist in the proliferation of MSCs and aid differentiation of cortex-derived NSCs [72]. However, due to the harsh external environment in the host body and lack of supportive substrates during transplantation, much of the transplanted cells lose its viability resulting in reduced therapeutic efficacy [73]. It has been reported that two dimensional thin film scaffolds, composed of biocompatible poly(acrylic acid) polymer grafted carbon nanotubes (CNTs), can selectively differentiate human embryonic stem cells into neuron cells while maintaining the viability of transplanted cells [74]. Even multiwalled carbon nanotube (MWNT) sheets showed to significantly enhance neural differentiation of hMSCs grown on the CNT sheets. Axon outgrowth was also controlled using nanoscale patterning of CNTs [75]. Recently, silk-CNT-based nanocomposite scaffolds are shown to protect and promote neuronal differentiation of hESCs [76]. Silks are natural polymers (protein) that have been widely used as biomaterials for many years. Fibroin, comprising the major portion of the silk protein fibre, consists of $90 \%$ of amino acids including glycine, alanine, and serine. Due to its strong mechanical and flexible nature in thin film form, biocompatibility, and in-vivobioresorbable properties, fibroin protein has been used as the building block for scaffolds. As confirmed by scanning electron microscope (Figure5 A-C), similar results were obtained with the developed silk-CNT scaffold where cells grown on the silk substrate exhibited denser complex three-dimensional axonal bundle networks as well as better spatial density distribution of the networks compared to other scaffolds. Overall, the silk-CNT nanocomposite provided an efficient three-dimensional supporting matrix for stem cell-derived neuronal transplants, offering a promising opportunity for nerve repair treatments for patients with neurological disorder. In-vitro analysis showed that $\beta$ - 
III tubulin, representing the mature differentiated neurons and nestin, representing the neuron precursors, were highly expressed in hESCs grown on the silk-CNT substrate compared to the expression level of cells grown on the control poly-L-ornithine substrate (figure 5D). In addition, hESCs cultured on the silk-CNT scaffold exhibited higher maturity along with dense axonal projections.
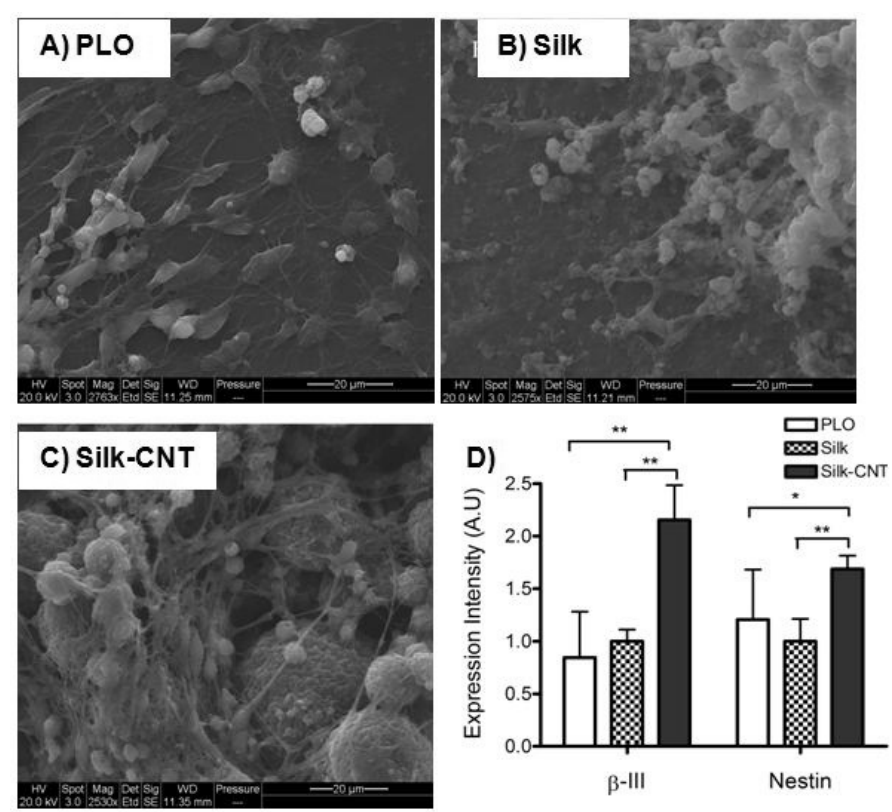

Figure 5. Scanning Electron Microscope images of hESCS on various substrates. SEM images of (A) cells cultured on PLO exhibiting a flat morphology and two-dimensional axonal connections, (B) cells cultured on silk scaffolds demonstrating three-dimensional structures and cell migration, and (C) cells cultured on silk-CNT scaffolds demonstrating three-dimensional axonal connections and silk-CNT matrix degradation. (D) Two neuronal markers ( $\beta$-III tubulin and nestin) were used to further determine the hESC differentiation efficiency. Expression intensity of $\beta$-III tubulin and nestin was observed with fluorescence microscopy. Silk-CNT scaffolds exhibited maximum $\beta$-III tubulin expression, while nestin expression exhibited a similar trend. ${ }^{*}=P<0.01$, and ${ }^{* *}=P<0.001$ [76]. Abbreviations: PLO, Poly-L-ornithine; hESCs, human embryonic stem cells; CNT, carbon nanotube.

\subsection{Carbon nanotubes for thermal destruction of tumors}

Tissues are known to be highly transparent to 700- to 1,100-nm near-infrared (NIR) light, whereas, SWNTs display strong optical absorbance in this special spectral window. When constantly absorb energy in NIR region, SWNTs emit heat [77]. Continuous heating leads to killing of the cells. SWNTs have been engineered with tumor recognition molecules for selective enteringcancer cells. Upon NIR radiation, the cancer cells were killed by thermal ablation [78-83]. Previous studies have shown that folic acid decorated SWNTs more effectively killed folate receptor positive cancer cells [83]; monoclonal antibody (mAb) against 
human CD22 conjugated SWNTsonly targeted CD22(+)CD25(-) Daudi cells; whereas, antiCD25 mAb coupled SWNTs only target CD22(-)CD25(+) activated peripheral blood mononuclear cells [81]. The thermal ablation effects can be combined with other therapies, eg. chemotherapy, by loading drugs on CNTs for synergic effect [32].

Tumors, in general, contain a small population of tumor initiating stem-like cells, termed as cancer stem cells. These cells are unmanageable by standard treatment modalities such as chemotherapy and radiotherapy and tend to persist after treatment [84]. Heat-based cancer treatments are increasingly becoming a potential alternative to approach this problem. Combining CNTs with such hyperthermia based therapies can further enhance its efficacy by simultaneously eliminating both the stem cells and bulk cancer cells that constitute a tumor. In fact, CNTs offer several properties that make them promising candidates for such thermal therapy. This includes their ability for thermal conductance and strong absorbance of electromagnetic radiation. It generates significant amounts of heat upon excitation with near-infrared light which is transparent to biological systems including skins. Such a photothermal effect can be employed to induce thermal cell death in a noninvasive manner. Thus, if CNTs can be localized to tumors, they can be stimulated with near-infrared radiation or radiofrequency energy to generate site-specific heat [85]. Preliminary in-vivo results show that a combination of multiwalled carbon nanotubes (MWNTs) and NIR can be useful for tumor regression and long-term survival in a mouse model [86]. Such CNT-mediated thermal therapy addresses the limitations of presently available medical strategies. This includes the minimally invasive site-specific heating which will greatly diminish the off-target toxicities, generation of uniform temperature distribution throughout the tumor mass by the activated CNTs, its compatibility with concurrent MRI temperature mapping techniques. It has also been recently reported that breast cancer stem cells, highly resistant to conventional thermal treatments, can be successfully treated with CNT-based photothermal therapies by promoting necrotic cell death [84]. Further studies in this direction shows that DNA-encased MWNTs are more efficient at converting NIR irradiation into heat compared to non-encased MWNTs and that this method can be effectively used in-vivo for the selective thermal ablation of cancer cells [87].

Glioblastomamultiforme is the most common and aggressive malignant primary brain tumor involving glial cells and accounting for a large percentage of brain and intracranial tumor $[88,89]$. It is also known for its recurrence and overall resistance to therapy. CD133+ stem cells occurring among GBM cells are responsible for such huge recurrence risk [90]. Research has been focused on developing strategies to efficiently deliver CNTs to these target sites, harboring CD133+ cancer stem cells [80]. In-vitro studies show that such targeted elimination of CD133 (+) cancer stem cells are possible by adding SWNTs functionalized with CD133 monoclonal antibody, followed by irradiation with NIR laser light. In a separate study, embryonic stem cells, once administered with MWNTs, have shown to induce an enhanced immune boost and provide subsequent anticancer protection in mice with colon cancer by suppressing the proliferation and development of malignant colon tumors [91]. 


\section{Carbon nanotubes for biomedical imaging and detection}

The well-ordered molecular structure attributes CNTs with multiple distinct optical properties, include strong NIR absorption, photoluminescence and Raman shift [92]. Structurally, SWNTs can be viewed as a cylinder rolled up by one layer of graphene sheet. TThere are infinite numbers of ways to roll a graphene sheet into a cylinder. Depending on different ways of wrapping, the particular nanotube could be metallic or semi-conductive. Individual semi-conductive SWNTs with appropriate chirality can generate a small band gap fluorescence of $1 \mathrm{eV}$, which corresponds to NIR range (900-1600 nm), where biological tissues have very low absorption, scattering, and autofluorescence, and therefore, are very useful for biological imaging. In the other side, the inherent graphene structure provides SWNTs with specific Raman scattering signature [93], which is strong enough for use in-vivo imaging. All these optical properties offer opportunities for SWNTs as contrast agents for near-infrared (NIR) photoluminescence imaging [94, 95], Raman imaging and optical absorption agent for photoacoustic imaging [96-98].

\subsection{Photoluminescence imaging}

NIR photoluminescence of micelle encapsulated SWNTs was firstly discovered by O.Connel et al [7]. The single-particle dispersion of individual nanotubes was prepared by ultrasonically agitating of raw SWNTs in SDS. The tube bundles, ropes, and residual catalyst were removed by ultracentrifugation, since the aggregation of nanotubes would quench fluorescence. One advantage of the photoluminescence of SWNTs over organic fluorescence dyes is that SWNTs have no apparent photobleaching, and therefore, the SWNTs could be a powerful tool for tracking changes in living system. Researchers have applied NIR photoluminescence of SWNTs for tracking endocytosis and exocytosis of SWNTs in NIH-3T3 cells in real time $[94,95]$. Moreover, conjugation of antibodies to SWNTs surface allowed specific cell targeting. They have shown that, with conjugation of anti-CD20, SWNTs selectively recognized CD20 cell surface receptor on B-cells with little binding to receptor negative T-cells. Similarly, with conjugation of Herceptin, SWNTs only recognize HER2/neu positive breast cancer cells. The selective binding of SWNTs was detected by intrinsic NIR photoluminescence of nanotubes. This technique allows deep tissue penetration and high-resolution intravital microscopy imaging of tumor vessels beneath thick skin $[99,100]$.

\subsection{Raman shift imaging}

Raman spectroscopy is a sensitive analytical tool for biological samples. It also has advantages of resistance to autofluorescence and photobleaching, high spatial resolution, and small sample size [101]. CNTs exhibit strong resonance Raman scattering with several distinctive scattering features including the radial breathing mode (RBM) and tangential mode (G-band) [93, 102]. Both RBM and G-band of CNTs are sharp and strong peaks, which can be easily distinguished from autofluorescence of tissue samples, Recently, Raman microspectroscopy of SWNTs has been applied for imaging of tissue samples, live cells, and small animal models [96-98]. Tumor targeted delivery by RGD peptide functionalized SWNTs has 
been investigated in murine tumor model [85]. Raman spectroscopy image of excised tissues confirmed efficient targeting of $\alpha v \beta 3$ integrin positive U87MG tumor by RGD [85]. This study also disclosed that CNTs have relatively long circulation time, and rapid renal clearance, which makes SWNTs an attractive diagnostic and therapeutic delivery vehicle. Zevaleta et al further developed a Raman microscope capable of noninvasive in-vivo evaluation of tumors in mice with RGD-labeled SWNTs. Using the dynamic Raman microscope, pharmacokinetics of SWNTs in the tumor was evaluated immediately following an intravenous injection of SWNTs. Raman spectral analysis revealed effectiveness of the RGD nanotubes to the integrin expressing U87MG tumor. The noninvasive Raman imaging results were compared with excised tissues and shows consistency [97].

\subsection{Photoacaustic imaging}

Photoacoustic imaging is an optical imaging technique that combines high optical absorption contrast with diffraction-limited resolution of ultrasonic imaging, which allows deeper tissues to be viewed in living subjects. In photoacoustic imaging, short pulses of stimulating radiation are absorbed by tissues, resulting a subsequent thermal expansion and ultrasonic emission that can be detected by highly sensitive piezoelectric devices. However, many diseases, for example cancer, in their early stages, do not exhibit a natural photoacoustic contrast, therefore administering an external photoacoustic contrast agent is necessary. Owing to the strong light absorption characteristic [77], the CNTs can be utilized as photoacoustic contrast agents. De la Zerdaet. al, in the first time, applied SWNTs for in-vivo imaging of tumors in mice [103]. In this study, SWNTs was surface modified by PL-PEG and further conjugated with cyclic RGD peptides for targeting $\alpha v \beta 3$ integrin on cancer cells. Intravenous injection of these cyclic RGD functionalized CNTs to mice bearing tumors showed eight times stronger photoacoustic signal in the tumors than mice injected with non-targeted CNTs. This study suggested that photoacoustic imaging using targeted SWNTs could contribute to non-invasive in-vivo cancer imaging [103]. Similarly, in another study, SWNTs functionalized with antibody against $\alpha v \beta 3$ integrin for photoacoustic imaging of human glioblastoma tumors in nude mice [104].

\section{Selected examples for preparation of carbon nanotube based therapeutics}

In the above CNTs for drug delivery section, we described the functionalization of CNTs with drugs or targeting molecules. These preparations are usually applied directly via intravenous delivery route, which is the most widely used route of drug administration. Alternative to the intravenous drug administration, some other routes of drug administrations are also important for certain specific applications. Different formulations of CNT-based therapeutics have also been developed to suit the specific routes of administration. Here, we report several novel cases of CNTs applications for oral and transdermal delivery routes. 


\subsection{Carbon nanotube based therapeutics delivery using artificial cells: oral delivery}

For many therapeutics, oral and targeted delivery are challenge. One way to deliver them at the targeted site is by novel methods of encapsulating them in polymeric artificial cells. Artificial cells are vesicles made by polymeric membranes. They can mimic certain functions of biological cells. The size of artificial cells ranged from nanometer to hundreds of micrometer [105]. The membranes of artificial cells are usually semi-permeable that allows for exchange of small molecules and prevention of passage of large substances across it. Up to date, artificial cells have been applied for encapsulation biologically active agents, including enzymes, hormones, drugs, even live bacteria cells for in-vivo delivery. Currently a couple of artificial cells have been applied clinically [106]. The advantages of artificial cells include protection of the cargos from immune elimination in the body, targeted delivery of cargos to desired sites and increasing cargo solubility [106]. In a first feasibility study, functionalized CNTs have been encapsulated in artificial cell made from biocompatible polymeric membrane for target specific delivery. The polymeric membrane was assembled with three layers of polymers, alginate-poly-L-lysine-alginate (APA), via electrostatic association (figure 6). Artificial cells protected CNT therapeutics from degradation by the harsh environments [107]. PH degradation profile of the polymeric membrane of artificial cells can be adjusted by composition of polymers, which allows the breakdown of the artificial cells and release of CNT therapeutics to desired sites. This system is ideal for oral delivery, and can be used for other delivery routes as well.

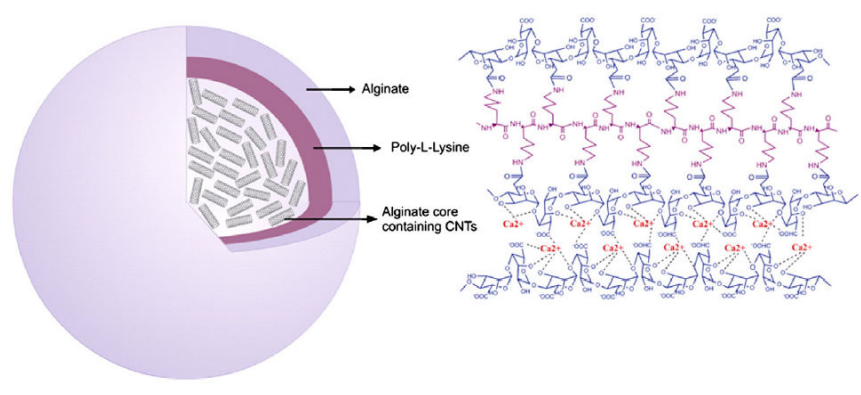

Figure 6. Alginate-poly-L-lysine-alginate (APA) microcapsules encapsulatingcarbon nanotubes. The calcium ions are responsible for cross-linking of the alginate monomeric units trapping the carbon nanotubes into the core of microcapsule [108].

\subsection{Carbon nanotube based membrane: transdermal drug delivery}

One of the most important areas of transdermal drug delivery (TDD) is in addiction treatment. Nicotine TDD has been widely used for smoking cessation programs. However, these traditional transdermal patches could not provide variable drug delivery rates. Some TDD has the capability to provide variable and programmable delivery rates, however, it needs a strong electric current across the human skin, which can cause serious skin irritation. Recently, the membranes prepared by functionalized CNTs have been employed in transder- 
mal drug delivery. It has been shown that this CNT-membrane was very effective for treatment of drug abuse and addiction [109]. To prepare the CNT-membrane, MWNTs were firstly functionalized with negatively charged molecule containing sulphonate $\left(-\mathrm{SO}^{3-}\right)$ to have a high charge density on the surface of CNTs, which is necessary to get efficient electro-osmosis pumping effect. By this functionalization strategy, CNT-membrane achieves dramatically fast flow through CNT cores, high charge density, and highly efficient electrophoretic pumping effect. These membranes were the integrated with a nicotine formulation to obtain switchable transdermal nicotine delivery rates on human skin (in vitro). The transdermal nicotine formulated CNT-membrane was able to successfully switch between high level and low level fluxes that coincide with therapeutic demand levels for nicotine cessation treatment. These programmable devices cause minimal skin irritation [109].

\section{Potentials and limitations of carbon nanotubes in medicine}

CNTs are being highly explored in the fields of targeted drug delivery, nanoscaffold for tissue engineering, biomedical imagining and detecting for disease treatment and health monitoring. The use of CNTs in drug delivery, detection and tissue engineering has shown the potentials to revolutionize medicine. CNTs affords for a large amounts of payloads for specific-targeting and drug delivery. With their intrinsic properties, the CNTs have potential for building-up multifunctional nanodevices for simultaneous therapeutic delivery and detection. Current cancer therapies (eg. radiation therapy, and chemotherapy) are usually painful and less efficient since they kill normal cells in addition to cancer cells, and therefore, producing adverse side effects and resistance. The CNT-based drug delivery systems have shown efficient tumor-targeting, and they can effectively kill cancer cells with a dosage lower than conventional drugs used, however significantly reduces side effects. Current CNTbased nanoscaffolds are very advantages for stem cells therapy in that they can modulate the stem cell growth and differentiation in a more controlled and desirable manner. Thus, CNTs have recently gained much interest in the field of medicine.

Although very useful, CNTs exist some limitations. Firstly, pristine CNTs, being metallic in their nature, are insoluble and they form large bundles or ropes in many solvents, including water and most solvents, so they cannot be used directly in biomedical applications. Much work has been done to prepare them for use in medicine. Secondly, the CNTs are not homogenous in their sizes (both diameters and lengths), which could be a problem for generation of reproducible results that allows evaluation of the biological activity relating to specific structures. Up to date, tremendous efforts have been put in surface functionalization of CNTs for use in medicine. This includes numerous effective methods for covalent or noncovalent modification of CNTs as to disperse them into aqueous solutions and to attach functional molecules for therapeutic applications. However, in terms of homegenecity of CNTs. Not much work has been done so far. We propose that attentions are needed to develop the methods for generation of CNTs with homogeneous size, which is very important for future clinical applications of CNT-based therapeutics. 
As a novel nanomaterial of great potentials in medicine, the toxicology of CNTs has received much attention in recent years. Pristine CNTs are very light powders and they can enter the body through inhalation via the respiratory tract, ingestion via the gastrointestinal tract or, dermal absorption via the skin. Following entering, CNTs distribute rapidly in the central and peripheral nervous system, lymphatic and blood circulation and potentially cause toxic effects in a variety of tissues and organs that they reach, such as heart, spleen, kidney, bone marrow and liver, etc. Toxicity of CNTs has been evaluated in a variety of cell or animal models for assessing pulmonary, dermal and immune effects. However, the published results have not led to any consensus on the toxicity profile of pristine or functionalized SWNTs and MWNTs. Some investigators reported that pristine SWNTs that were purified by acid treatment demonstrated no acute toxicity, as opposed to non-purified CNTs, however, they induced reactive oxygen species (ROS) in human lung carcinoma epithelial A549 cells and NR8383 cells [110]. Others demonstrated that pristine SWNTs, either acid-treated or non-treated, were capable of increasing chromosome and DNA damage, and oxidative stress in macrophage cell lines [111, 112].

In contrast to raw or acid treated CNTs, the well-dispersed CNTs with high levels of surface functionalization can reduce the toxicity of MWNTs. One study demonstrated that taurineMCWNTs in low and medium doses induced slight and recoverable pulmonary inflammation in mice, and are less toxic than raw MCWNTs [113]. This is supported by other studies indicating that the damage caused by non-PEGylated MWNTs is slightly more severe than that of PEGylated MWNTs [114]. Furthermore, administrations of high doses of PL-PEG functionalized SWNTs following intravenous injection did not lead to acute or chronic toxicity in nude mice, albeit SWNTs persisted within liver and spleen macrophages for 4 months in mice without apparent toxicity [115] and the SWNTs-PL-PEG were excreted from mice via the biliary and renal pathways [116]. It is hypothesized that the van der Waals forces on the surfaces of pristine CNTs cause hydrophobic interactions between CNTs, resulting in aggregation and network formation, which further induce prolonged toxicity. Thus, functionalization of CNTs overcomes the aggregate-forming surface properties of CNTs, and therefore, reduces toxicity.

\section{Conclusions:}

CNTs have exhibited diverse physical, chemical and mechanical properties suitable for a variety of applications. In last decade, biomedical applications of CNTs have undergone rapid progress. Their unique properties, such as, ultrahigh surface area, high aspect ratio, distinct optical properties have been applied to develop innovative, multi-functional CNT-based nanodevices for broad applications. This chapter have described the chemical and physical methods to prepare CNTs for used in medicine. With these methods, targeting molecules are attached on CNTs for targeted drug delivery, selective imaging, and other therapies. As a new type of nanomaterial, the toxicity of CNTs has been extensively investigated. To date, tremendous toxicity studies on CNTs have been published. However, the published data are inconsistent. The reason is that CNTs used in these studies vary in dispersion status, size 
and length of tubes, metal impurities and functionalization methods etc. Moreover, different analysis methods used in the evaluation CNTs toxicity studies also cause disparities. Despite these disparities, there is a broad agreement that well-dispersed CNTs have little or no toxicity both in-vitro and in-vivo, and therefore are useful for biomedical applications. Finally, an urgent need has been proposed for long-term studies on the absorption, deposition, metabolism and excretion (ADME) of CNTs. Only after the uncertainty on CNT toxicity is resolved, the CNT-based therapeutics can be possible applied clinically.

\section{Acknowledgements}

This work is partially supported by research grant to Satya Prakash from Canadian Institutes of Health Research (CIHR) (MOP 93641). W. Shao and L. Rodes acknowledges Doctor Training Award from Fonds de Research Sante (FRSQ). A. Paul acknowledges Post-Doctoral Award from FRSQ.

\section{Author details}

Wei Shao ${ }^{1}$, Paul Arghya ${ }^{1}$, Mai Yiyong ${ }^{2}$, Laetitia Rodes ${ }^{1}$ and Satya Prakash ${ }^{1 *}$

*Address all correspondence to: satya.prakash@mcgill.ca

1 Biomedical Technology and Cell Therapy Research Laboratory, Department of Biomedical Engineering and Artificial cells and Organs Research Centre, Faculty of Medicine, McGill University, Canada

2 Department of Chemistry, McGill University, Canada

\section{References}

[1] Chen, Z., et al. (2011). Single-walled carbon nanotubes as optical materials for biosensing. Nanoscale, 3(5), 1949-1956.

[2] Bekyarova, E., et al. (2005). Electronic properties of single-walled carbon nanotube networks. Journal of the American Chemical Society, 5990-5995.

[3] Kostarelos, K., et al. (2007). Cellular uptake of functionalized carbon nanotubes is independent of functional group and cell type. Nat Nanotechnol, 2(2), 108-113.

[4] Ando, Y. (2010). Carbon nanotube: the inside story. J Nanosci Nanotechnol, 10(6), 3726-3738. 
[5] Sang Won Kim, T.K, Yern Seung, Kim, Hong Soo, Choi, Hyeong, Jun Lim, Seung Jae, Yang, \& Chong Rae, Park. (2012). Surface modifications for the effective dispersion of carbon nanotubes in solvents and polymers. Carbon, 50(1), 30.

[6] Tasis, D., et al. (2003). Soluble carbon nanotubes. Chemistry, 9(17), 4000-4008.

[7] O'Connell, M. J., et al. (2002). Band gap fluorescence from individual single-walled carbon nanotubes. Science, 297(5581), 593-596.

[8] Valerie, C., Moore, M. S. S., Erik, H., Haroz, Robert. H., \& Hauge, Richard E. (2003). Smalley, Individually Suspended Single-Walled Carbon Nanotubes in Various Surfactants. Nano Lett, 3(10), 3.

[9] Strano, M. S., et al. (2003). The role of surfactant adsorption during ultrasonication in the dispersion of single-walled carbon nanotubes. J Nanosci Nanotechnol, 3(1-2), 81-86.

[10] Liu, Z., et al. (2009). Preparation of carbon nanotube bioconjugates for biomedical applications. Nat Protoc, 4(9), 1372-1382.

[11] Fukushima, T, et al. (2003). Molecular ordering of organic molten salts triggered by single-walled carbon nanotubes. Science, 300(5628), 2072-2074.

[12] Dong, B., et al. (2011). Dispersion of carbon nanotubes by carbazole-tailed amphiphilic imidazolium ionic liquids in aqueous solutions. Journal of colloid and interface science, 190-195.

[13] Fu, Q., \& Liu, J. (2005). Effects of ionic surfactant adsorption on single-walled carbon nanotube thin film devices in aqueous solutions. Langmuir : the ACS journal of surfaces and colloids, 1162-1165.

[14] Tasis, D., et al. (2006). Chemistry of carbon nanotubes. Chem Rev, 106(3), 1105-1136.

[15] Petrov, P., et al. (2003). Noncovalent functionalization of multi-walled carbon nanotubes by pyrene containing polymers. Chem Commun (Camb) [23], 2904-2905.

[16] Li, H., et al. (2004). Selective interactions of porphyrins with semiconducting singlewalled carbon nanotubes. Journal of the American Chemical Society, 1014-1015.

[17] Xianbao Wang, Y.L, Wenfeng, Qiu, \& Daoben, Zhu. (2002). Immobilization of tetratert-butylphthalocyanines on carbon nanotubes: a first step towards the development of new nanomaterials. J. Mater. Chem, 12(6), 3.

[18] Kang, Y. K., et al. (2009). Helical wrapping of single-walled carbon nanotubes by water soluble poly(p-phenyleneethynylene). Nano letters, 9(4), 1414-1418.

[19] Zheng, M., et al. (2003). DNA-assisted dispersion and separation of carbon nanotubes. Nature materials, 338-342.

[20] Hu, H., et al. (2003). Nitric Acid Purification of Single-Walled Carbon Nanotubes. The Journal of Physical Chemistry B, 107(50), 13838-13842. 
[21] Karousis, N., Tagmatarchis, N., \& Tasis, D. (2010). Current progress on the chemical modification of carbon nanotubes. Chem Rev, 110(9), 5366-5397.

[22] Georgakilas, V., et al. (2002). Amino acid functionalisation of water soluble carbon nanotubes. Chem Commun (Camb) [24], 3050-3051.

[23] Ojima, I. (2008). Guided molecular missiles for tumor-targeting chemotherapy--case studies using the second-generation taxoids as warheads. Accounts of chemical research, 108-119.

[24] Krauss, W. C., et al. (2000). Emerging antibody-based HER2 (ErbB-2/neu) therapeutics. Breast Dis, 11, 113-124.

[25] Abramson, J. S., \& Shipp, M. A. (2005). Advances in the biology and therapy of diffuse large B-cell lymphoma: moving toward a molecularly targeted approach. Blood, 1164-1174.

[26] Garanger, E., Boturyn, D., \& Dumy, P. (2007). Tumor targeting with RGD peptide ligands-design of new molecular conjugates for imaging and therapy of cancers. Anticancer Agents Med Chem, 7(5), 552-558.

[27] Corti, A., \& Curnis, F. (2011). Tumor Vasculature Targeting Through NGR PeptideBased Drug Delivery Systems. Current Pharmaceutical Biotechnology, 1128-1134.

[28] Lu, Y., \& Low, P. S. (2002). Folate-mediated delivery of macromolecular anticancer therapeutic agents. Advanced drug delivery reviews, 675-693.

[29] Hermanson, G. T. (2008). Bioconjugate Techniques,2nd Edition. Academic Press, Inc., 1202, pages.

[30] Liu, Z., et al. (2007). Supramolecular chemistry on water-soluble carbon nanotubes for drug loading and delivery. ACS nano, 50-56.

[31] Ji, Z., et al. (2012). Targeted therapy of SMMC-7721 liver cancer in vitro and in vivo with carbon nanotubes based drug delivery system. Journal of colloid and interface science, 143-149.

[32] Wang, L., et al. (2011). Synergistic enhancement of cancer therapy using a combination of docetaxel and photothermal ablation induced by single-walled carbon nanotubes. Int J Nanomedicine, 6, 2641-2652.

[33] Taghdisi, S. M., et al. (2011). Reversible targeting and controlled release delivery of daunorubicin to cancer cells by aptamer-wrapped carbon nanotubes. European journal of pharmaceutics and biopharmaceutics official journal of Arbeitsgemeinschaft fur Pharmazeutische Verfahrenstechnik e., 77, 200-206.

[34] Lu, Y. J., et al. (2012). Dual targeted delivery of doxorubicin to cancer cells using folate-conjugated magnetic multi-walled carbon nanotubes. Colloids Surf B Biointerfaces, $89,1-9$. 
[35] Lay, C. L., et al. (2010). Delivery of paclitaxel by physically loading onto poly(ethylene glycol) (PEG)-graft-carbon nanotubes for potent cancer therapeutics. Nanotechnology, 21(6), 065101.

[36] Bhirde, A. A., et al. (2009). Targeted killing of cancer cells in vivo and in vitro with EGF-directed carbon nanotube-based drug delivery. ACS nano, 307-316.

[37] Liu, Z., et al. (2008). Drug delivery with carbon nanotubes for in vivo cancer treatment. Cancer research, 68(16), 6652-6660.

[38] Tsavaris, N. B., \& Kosmas, C. (1998). Risk of severe acute hypersensitivity reactions after rapid paclitaxel infusion of less than 1-h duration. Cancer chemotherapy and pharmacology, 509-511.

[39] Sendo, T., et al. (2005). Incidence and risk factors for paclitaxel hypersensitivity during ovarian cancer chemotherapy. Cancer chemotherapy and pharmacology, 91-96.

[40] Zhang, X., et al. (2009). Targeted delivery and controlled release of doxorubicin to cancer cells using modified single wall carbon nanotubes. Biomaterials, 30(30), 6041-6047.

[41] Yang, F, et al. (2011). Magnetic functionalised carbon nanotubes as drug vehicles for cancer lymph node metastasis treatment. Eur J Cancer, 47(12), 1873-1882.

[42] Dhar, S., et al. (2008). Targeted single-wall carbon nanotube-mediated Pt(IV) prodrug delivery using folate as a homing device. Journal of the American Chemical Society, 130(34), 11467-11476.

[43] Sun, X., \& Zhang, N. (2010). Cationic polymer optimization for efficient gene delivery. Mini reviews in medicinal chemistry, 108-125.

[44] Shao, W., et al. (2012). A novel polyethyleneimine-coated adeno-associated virus-like particle formulation for efficient siRNA delivery in breast cancer therapy: preparation and in vitro analysis. Int J Nanomedicine, 7, 1575-1586.

[45] Yang, F., et al. (2009). Pilot study of targeting magnetic carbon nanotubes to lymph nodes. Nanomedicine, 317-330.

[46] Nunes, A, et al. (2010). Hybrid polymer-grafted multiwalled carbon nanotubes for in vitro gene delivery. Small, 6(20), 2281-2291.

[47] Pantarotto, D., et al. (2004). Functionalized carbon nanotubes for plasmid DNA gene delivery. Angew Chem Int Ed Engl, 43(39), 5242-5246.

[48] Singh, R., et al. (2005). Binding and condensation of plasmid DNA onto functionalized carbon nanotubes: toward the construction of nanotube-based gene delivery vectors. Journal of the American Chemical Society, 4388-4396.

[49] Wang, X., et al. (2009). Knocking-down cyclin A(2) by siRNA suppresses apoptosis and switches differentiation pathways in K562 cells upon administration with doxorubicin. PloS one, 4(8), e6665. 
[50] Zhang, Z., et al. (2006). Delivery of telomerase reverse transcriptase small interfering RNA in complex with positively charged single-walled carbon nanotubes suppresses tumor growth. Clinical cancer research : an official journal of the American Association for Cancer Research, 4933-4939.

[51] Wang, X., Ren, J., \& Qu, X. (2008). Targeted RNA interference of cyclin A2 mediated by functionalized single-walled carbon nanotubes induces proliferation arrest and apoptosis in chronic myelogenous leukemia K562 cells. ChemMedChem, 3(6), 940-945.

[52] Yang, R., et al. (2006). Single-walled carbon nanotubes-mediated in vivo and in vitro delivery of siRNA into antigen-presenting cells. Gene therapy, 13(24), 1714-1723.

[53] Liu, Y., et al. (2005). Polyethylenimine-grafted multiwalled carbon nanotubes for secure noncovalent immobilization and efficient delivery of DNA. Angew Chem Int Ed Engl, 44(30), 4782-4785.

[54] Liu, Z, et al. (2007). siRNA delivery into human T cells and primary cells with carbon-nanotube transporters. Angew Chem Int Ed Engl, 46(12), 2023-2027.

[55] Kam, N. W., Liu, Z., \& Dai, H. (2005). Functionalization of carbon nanotubes via cleavable disulfide bonds for efficient intracellular delivery of siRNA and potent gene silencing. Journal of the American Chemical Society, 127(36), 12492-12493.

[56] Bartholomeusz, G., et al. (2009). In Vivo Therapeutic Silencing of Hypoxia-Inducible Factor 1 Alpha (HIF-1alpha) Using Single-Walled Carbon Nanotubes Noncovalently Coated with siRNA. Nano Res, 2(4), 279-291.

[57] Paul, A., et al. (2009). Microencapsulated stem cells for tissue repairing: implications in cell-based myocardial therapy. Regen Med, 4(5), 733-745.

[58] Harrison, B. S., \& Atala, A. (2007). Carbon nanotube applications for tissue engineering. Biomaterials, 28(2), 344-353.

[59] Chao, T. I., et al. (2010). Poly(methacrylic acid)-grafted carbon nanotube scaffolds enhance differentiation of hESCs into neuronal cells. Adv Mater, 22(32), 3542-3547.

[60] Mooney, E, et al. (2008). Carbon nanotubes and mesenchymal stem cells: biocompatibility, proliferation and differentiation. Nano Lett, 8(8), 2137-2143.

[61] Martinelli, V, et al. (2012). Carbon nanotubes promote growth and spontaneous electrical activity in cultured cardiac myocytes. Nano Lett, 12(4), 1831-1838.

[62] Stout, D. A., Basu, B., \& Webster, T. J. (2011). Poly(lactic-co-glycolic acid): carbon nanofiber composites for myocardial tissue engineering applications. Acta Biomater, 7(8), 3101-3112.

[63] Oh, S, et al. 2009. Stem cell fate dictated solely by altered nanotube dimension. Proc Natl Acad Sci U S A, 106(7), 2130-2135.

[64] Tran, P. A., Zhang, L., \& Webster, T. J. (2009). Carbon nanofibers and carbon nanotubes in regenerative medicine. Adv Drug Deliv Rev, 61(12), 1097-1114. 
[65] Park, J., et al. (2012). Synergistic control of mesenchymal stem cell differentiation by nanoscale surface geometry and immobilized growth factors on $\mathrm{TiO} 2$ nanotubes. Small, 8(1), 98-107.

[66] Lai, M., et al. (2011). Surface functionalization of $\mathrm{TiO} 2$ nanotubes with bone morphogenetic protein 2 and its synergistic effect on the differentiation of mesenchymal stem cells. Biomacromolecules, 12(4), 1097-1105.

[67] Hu, Y., et al. (2012). TiO2 nanotubes as drug nanoreservoirs for the regulation of mobility and differentiation of mesenchymal stem cells. Acta Biomater, 8(1), 439-448.

[68] Connick, P., Patani, R., \& Chandran, S. (2011). Stem cells as a resource for regenerative neurology. Pract Neurol, 11(1), 29-36.

[69] Levenberg, S., et al. (2005). Neurotrophin-induced differentiation of human embryonic stem cells on three-dimensional polymeric scaffolds. Tissue Eng, 11(3-4), 506-512.

[70] Jan, E., \& Kotov, N. A. (2007). Successful differentiation of mouse neural stem cells on layer-by-layer assembled single-walled carbon nanotube composite. Nano Lett, 7(5), 1123-1128.

[71] Mazzatenta, A., et al. (2007). Interfacing neurons with carbon nanotubes: electrical signal transfer and synaptic stimulation in cultured brain circuits. J Neurosci, 27(26), 6931-6936.

[72] Nho, Y., et al. (2010). Adsorption of mesenchymal stem cells and cortical neural stem cells on carbon nanotube/polycarbonate urethane. Nanomedicine (Lond, 5(3), 409-417.

[73] Zhang, S. C., et al. (2001). In vitro differentiation of transplantable neural precursors from human embryonic stem cells. Nat Biotechnol, 19(12), 1129-1133.

[74] Chao, T. I., et al. (2009). Carbon nanotubes promote neuron differentiation from human embryonic stem cells. Biochem Biophys Res Commun, 384(4), 426-430.

[75] Kim, J. A., et al. (2012). Regulation of morphogenesis and neural differentiation of human mesenchymal stem cells using carbon nanotube sheets. Integr Biol (Camb, 4(6), 587-594.

[76] Chen, C. S., et al. (2012). Human stem cell neuronal differentiation on silk-carbon nanotube composite. Nanoscale Res Lett, 7(1), 126.

[77] Berber, S., Kwon, Y. K., \& Tomanek, D. (2000). Unusually high thermal conductivity of carbon nanotubes. Phys Rev Lett, 84(20), 4613-4616.

[78] Marches, R., et al. (2009). Specific thermal ablation of tumor cells using single-walled carbon nanotubes targeted by covalently-coupled monoclonal antibodies. Int J Cancer, 125(12), 2970-2977.

[79] Gannon, C. J., et al. (2007). Carbon nanotube-enhanced thermal destruction of cancer cells in a noninvasive radiofrequency field. Cancer, 110(12), 2654-2665. 
[80] Wang, C. H., et al. (2011). Photothermolysis of glioblastoma stem-like cells targeted by carbon nanotubes conjugated with CD133 monoclonal antibody. Nanomedicine, 7(1), 69-79.

[81] Chakravarty, P., et al. (2008). Thermal ablation of tumor cells with antibody-functionalized single-walled carbon nanotubes. Proceedings of the National Academy of Sciences of the United States of America, 105(25), 8697-8702.

[82] Xiao, Y., et al. (2009). Anti-HER2 IgY antibody-functionalized single-walled carbon nanotubes for detection and selective destruction of breast cancer cells. BMC cancer, $9,351$.

[83] Kam, N. W., et al. (2005). Carbon nanotubes as multifunctional biological transporters and near-infrared agents for selective cancer cell destruction. Proceedings of the National Academy of Sciences of the United States of America, 102(33), 11600-11605.

[84] Burke, A. R., et al. (2012). The resistance of breast cancer stem cells to conventional hyperthermia and their sensitivity to nanoparticle-mediated photothermal therapy. Biomaterials, 33(10), 2961-2970.

[85] Liu, Z., et al. (2007). In vivo biodistribution and highly efficient tumour targeting of carbon nanotubes in mice. Nat Nanotechnol, 2(1), 47-52.

[86] Burke, A., et al. (2009). Long-term survival following a single treatment of kidney tumors with multiwalled carbon nanotubes and near-infrared radiation. Proc Natl Acad Sci U S A, 106(31), 12897-12902.

[87] Ghosh, S., et al. (2009). Increased heating efficiency and selective thermal ablation of malignant tissue with DNA-encased multiwalled carbon nanotubes. ACS Nano, 3(9), 2667-2673.

[88] Singh, S. K., et al. (2003). Identification of a cancer stem cell in human brain tumors. Cancer Res, 63(18), 5821-5828.

[89] Lanzetta, G., et al. (2003). Temozolomide in radio-chemotherapy combined treatment for newly-diagnosed glioblastoma multiforme: phase II clinical trial. Anticancer Res, 23(6D), 5159-5164.

[90] Zeppernick, F., et al. (2008). Stem cell marker CD133 affects clinical outcome in glioma patients. Clin Cancer Res, 14(1), 123-129.

[91] Mocan, T, \& Iancu, C. (2011). Effective colon cancer prophylaxis in mice using embryonic stem cells and carbon nanotubes. Int J Nanomedicine, 6, 1945-1954.

[92] Xie, S. S., et al. (2000). Mechanical and physical properties on carbon nanotube. Journal of Physics and Chemistry of Solids, 61(7), 1153-1158.

[93] Tasis, D., et al. (2008). Diameter-selective solubilization of carbon nanotubes by lipid micelles. J Nanosci Nanotechnol, 8(1), 420-423. 
[94] Strano, M. S., \& Jin, H. (2008). Where is it heading? Single-particle tracking of singlewalled carbon nanotubes. ACS nano, 2(9), 1749-1752.

[95] Jin, H., Heller, D. A., \& Strano, M. S. (2008). Single-particle tracking of endocytosis and exocytosis of single-walled carbon nanotubes in NIH-3T3 cells. Nano letters, 8(6), 1577-1585.

[96] Keren, S., et al. (2008). Noninvasive molecular imaging of small living subjects using Raman spectroscopy. Proceedings of the National Academy of Sciences of the United States of America, 105(15), 5844-5849.

[97] Zavaleta, C., et al. (2008). Noninvasive Raman spectroscopy in living mice for evaluation of tumor targeting with carbon nanotubes. Nano letters, 8(9), 2800-2805.

[98] Liu, Z., et al. (2010). Multiplexed Five-Color Molecular Imaging of Cancer Cells and Tumor Tissues with Carbon Nanotube Raman Tags in the Near-Infrared. Nano Res, 3(3), 222-233.

[99] Welsher, K., et al. (2008). Selective probing and imaging of cells with single walled carbon nanotubes as near-infrared fluorescent molecules. Nano letters, 8(2), 586-590.

[100] Welsher, K., et al. (2009). A route to brightly fluorescent carbon nanotubes for nearinfrared imaging in mice. Nat Nanotechnol, 4(11), 773-780.

[101] Hanlon, E. B., et al. (2000). Prospects for in vivo Raman spectroscopy. Physics in medicine and biology, 45(2), R1-59.

[102] Karmakar, A., et al. (2011). Raman spectroscopy as a detection and analysis tool for in vitro specific targeting of pancreatic cancer cells by EGF-conjugated, single-walled carbon nanotubes. J Appl Toxicol.

[103] De la Zerda, A., et al. (2008). Carbon nanotubes as photoacoustic molecular imaging agents in living mice. Nat Nanotechnol, 3(9), 557-562.

[104] Xiang, L., et al. (2009). Photoacoustic molecular imaging with antibody-functionalized single-walled carbon nanotubes for early diagnosis of tumor. J Biomed Opt, 14(2), 021008.

[105] Chang, T. M. (2004). Artificial cell bioencapsulation in macro, micro, nano, and molecular dimensions: keynote lecture. Artificial cells, blood substitutes, and immobilization biotechnology, 32(1), 1-23.

[106] Prakash, S. (2007). Artificial cells, cell engineering and therapy:. Woodhead.

[107] Kulamarva, A., et al. (2009). Microcapsule carbon nanotube devices for therapeutic applications. Nanotechnology, 20(2), 025612.

[108] Prakash, S., et al. (2011). Polymeric nanohybrids and functionalized carbon nanotubes as drug delivery carriers for cancer therapy. Advanced drug delivery reviews, 63(14-15), 1340-1351. 
[109] $\mathrm{Wu}$, J., et al. (2010). Programmable transdermal drug delivery of nicotine using carbon nanotube membranes. Proceedings of the National Academy of Sciences of the United States of America, 107(26), 11698-11702.

[110] Pulskamp, K., Fau-Diabate, S., Diabate, H. F., Fau-Krug, S., \& Krug, H. F. Carbon nanotubes show no sign of acute toxicity but induce intracellular reactive oxygen species in dependence on contaminants. 0378-4274, Print.

[111] Migliore, L., Fau-Saracino, D., et al. Carbon nanotubes induce oxidative DNA damage in RAW 264.7 cells. 1098-2280, Electronic.

[112] Kagan Ve Fau- Tyurina, Y.Y, et al. Direct and indirect effects of single walled carbon nanotubes on RAW 264.7 macrophages: role of iron. 0378-4274, Print.

[113] Wang, X., Fau-Zang, J. J., et al. Pulmonary toxicity in mice exposed to low and medium doses of water-soluble multi-walled carbon nanotubes. 1533-4880, Print.

[114] Zhang, D., Fau-Deng, X., et al. Long-term hepatotoxicity of polyethylene-glycol functionalized multi-walled carbon nanotubes in mice. 1361-6528, Electronic.

[115] Schipper, Ml., Fau-Nakayama-Ratchford, N., et al. A pilot toxicology study of singlewalled carbon nanotubes in a small sample of mice. 1748-3395, Electronic.

[116] Liu, Z., Fau-Davis, C., et al. Circulation and long-term fate of functionalized, biocompatible single-walled carbon nanotubes in mice probed by Raman spectroscopy. 1091-6490, Electronic. 
